\title{
An early warning monitoring system for quality control in a water distribution network
}

\author{
R. Guercio \& E. Di Ruzza \\ Department of Hydraulic, Transportation and Roads, \\ Faculty of Engineering, University of Rome "La Sapienza", Italy
}

\begin{abstract}
The goal of an early warning water monitoring system (EWWMS) for quality control in a water distribution network is to identify low probability/high impact contamination events in source water or distribution systems. It should be able to detect not only intentional contamination, but also contaminants introduced accidentally or natural occurrences. Firstly, to ensure the full protection of drinking water, a technology- based EWWMS should be one component of the program. While laboratory technology exists to measure a wide range of contaminants, it's not the same for on-line and real time technology. A number of research projects are investigating rapid and on-line monitoring technologies, including biosensors and biochips, fiber optics and microelectronics. In most cases the performances of these systems has not been fully characterized. In this paper, a submergible UV/VIS spectrometer has been extensively tested in laboratory experiments, with the aim to check its limits of sensitivity, its ability to identify and quantify specific contaminants and its rate of false positive/negative.

Keywords: early warning water monitoring system; drinking water distribution system control; spectroscopy; on-line monitoring, tracer test, contamination.
\end{abstract}

\section{Introduction}

The goal of an EWWMS is to identify reliably low probability/high impact contamination events in source water or distribution systems. Monitoring water quality in real time allows an effective local response that minimizes the adverse impacts that may result from the event $[1,14]$. Other desirable features of an EWWMS include: affordable cost, low skill and training, coverage of all 
potential threats, ability to identify source, enough sensitivity to quality changes at regulatory levels, minimal false positive or negative responses, robustness, reproducible and verifiable results, availability of remote operation. Analytical chemical methods are usually target oriented; in other words, these methods can detect only a specific compound or a range of compounds having similar properties. Furthermore, analytical-chemical identification does not give information about bioavailability and possible toxic effects, especially from mixtures of compounds. On the other hand, changes in the behaviour or properties of on-line biological early warning systems may indicate the sudden occurrence of a pollutant not detected in conventional, analytical warning systems. [4-6]

The EWWMS here described was developed and field applied to ensure water quality protection for one of the inlet points of the Acquedotto del Ruzzo water distribution system, which mainly supplies drinking water to Teramo city (about 500.000 people) in Abruzzo region, Italy. The objective of this monitoring system is to check accidental spills or contaminations coming from temporary on-working activities carried on in one of the two car tunnels placed just above the water source in the Gran Sasso Mountain, and to detect potential accidental spills coming from the National Institute of Nuclear Physics (INFN), also placed into the Gran Sasso Mountain, that is one of the largest world underground research laboratories for Nuclear Physics and cosmic research.

Owing to the relevance for supply uses of the water flowing around the highway galleries and due to recent and limited time spills of contaminant from INFN laboratory, which were not detected by the current conventional monitoring system, a new advanced real time EWWM system was designed and installed in some critical points of the water supply pipe, in order to ensure the best possible level of security and to quickly detect possible water contaminations.

\section{Methods}

The new EWWMS was designed and developed by Systea Srl (Italy) to be completely modular, expandable and very flexible in its use, in order to be easily adapted and installed in different monitoring sites during the experimentation phase and the operative survey activity.

The basic layout of the EWWMS is composed by:

- a $220 \mathrm{Vac} / 12 \mathrm{Vcc}$ external power supply, mounted in a IP-65 wall mounting box;

- a local control unit made by a wall mounting IP65 plastic box, which contains the industrial PC and the I/O devices, the 12 Vec power supply with back-up batteries and any auxiliary hardware device;

- a multiparametric probe (YSI 6820, YSI Environmental Inc., USA) to measure standard physical-chemical parameters in water (temperature, $\mathrm{pH}$, conductivity, turbidity) which can be placed, according to the specific site requirement, directly in the water source or working off-line ; 
- an in-situ UV spectrometer (Spectro::lyzer, s::can Messtechnik GmbH, Austria) mounted in a special horizontal flow-through cell, to detect organic compounds;

- a water flow sensor, to be chosen according to the different installation site and application specification (Argonaut YSI/Sontek, (USA), electromagnetic flow sensors, in-tube mounting type ultrasonic sensors);

- a $500 \mathrm{ml}, 8$ bottles automatic sampler, specifically designed in a IP-65 wall mounting layout, for the collection of water samples in presence of threshold alarms detected by the on-line measuring devices.
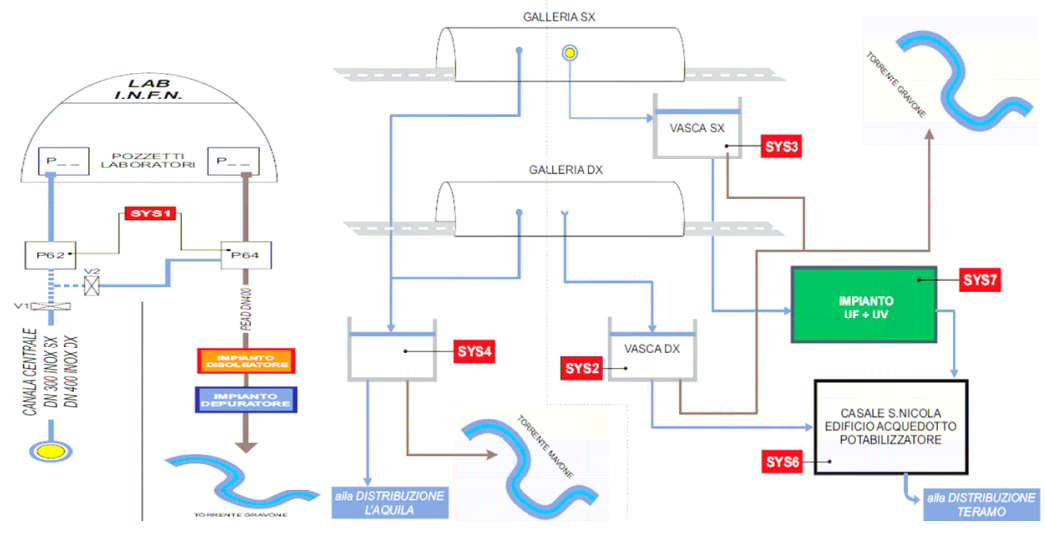

Figure 1: On- line monitoring system and sensors locations.

Depending on the specific installation site, each monitoring station is connected with an external Web server through the use of dedicated TCP-IP communication lines, automatically connected to the Internet through analogical or digital modems, private DSL line coupled with an ISDN external line, using the actual cheaper and very flexible routing technologies.

Each monitoring station is also connected to an industrial GSM device to send to operator's and technician's mobile phones Short Messages, informing them of any alarms and parameter's thresholds overlaps, which will cause a reaction on the system with automatic closure of hydraulic valves mounted on the tubing, to quickly redirect the water to be discharged in spite of being introduced in the potable water distribution system.

The tested submersible UV/Vis spectrometer measures absorbance of ultraviolet light from 200 to $400 \mathrm{~nm}$. The instrument is built as a compact submersible sensor enabling measurements of UV/Vis spectra; The results of the measurements are recorded and displayed in real-time, with a single measurement typically taking 45 seconds.

The instrument is a 2-beam, $256 \mathrm{pixel}, \mathrm{UV} / \mathrm{V}$ is spectrophotometer, with a Xenon lamp as a light source. By recording the light absorption in a sample 
between $200-400 \mathrm{~nm}$, the probe is capable of determining more than simply a fingerprint spectrum; besides this fingerprint, the values for a number of specific parameters are calculated from the data contained in the fingerprint, using algorithms provided by $\mathrm{S}:$ :can $[8,9]$.

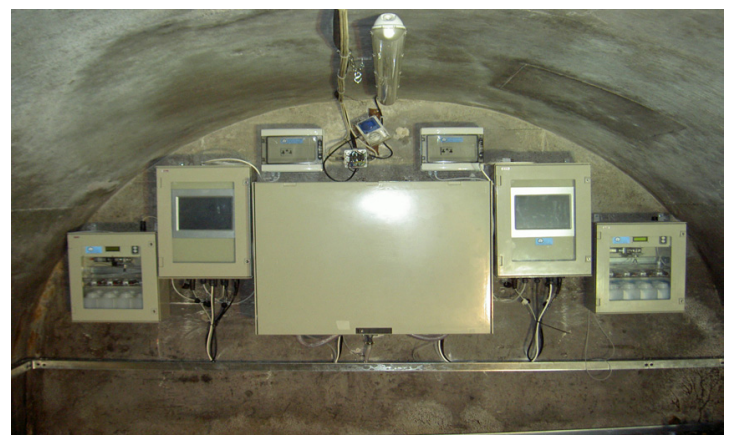

Figure 2: $\quad$ Installation of EWWS monitoring station.

\section{Results}

\subsection{Laboratory tests}

The parameter deducted from the fingerprint, using the software provided by Scan, are: Turbidity, TOC, DOC, Nitrate, SAK254.

We could besides monitor specific compounds, once their fingerprint is known and recorded on the probe; for this purpose we decided to use it to monitor also Benzene and Tri-methyl- benzene.

The first one because it could show pollution contamination from the high way road inside the tunnel. The second one because of possible accidental spills coming from the underground laboratory.

The calibration curves were determined using local calibration provided by the software. A global calibration is also available but for particular water composition, like drinking water, the local one is more accurate. The measurements were performed with both drinking and distilled water to appreciate the effects of the water matrix on the measurements. We performed cross sensitivity test between nitrate and benzene and between nitrates and Trimethyl-benzene to study how they interact with each other. For calibration, samples with known concentration were measured and we determined the accuracy of the instruments, the higher and lower detection limits. Figures 3, 4, 5 , show the local calibration curves determined for the all the parameters of the probe.

The nitrate could be detected in drinking water down to $0,1 \mathrm{ppm}$ NO3 -Neq. Below this concentration, the deviation from background spectra became too small for detection. The upper level of detection was $11 \mathrm{ppm}$, even if the calibration curve results linear until 6 ppm; over that limit the correlation became polynomial. 


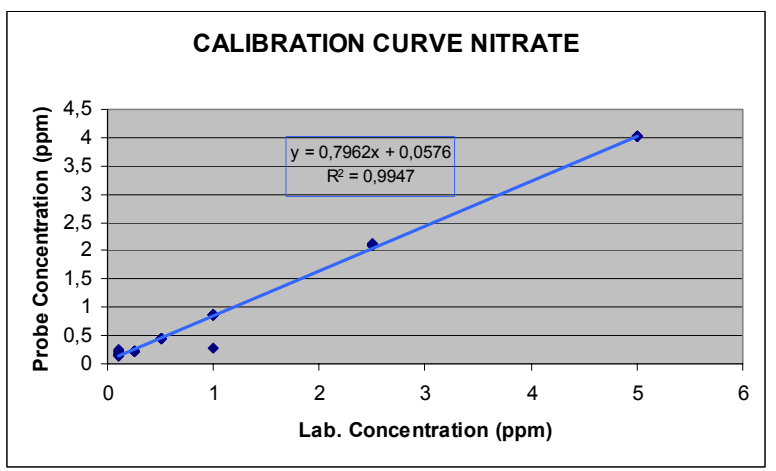

Figure 3: Nitrates calibration curve.

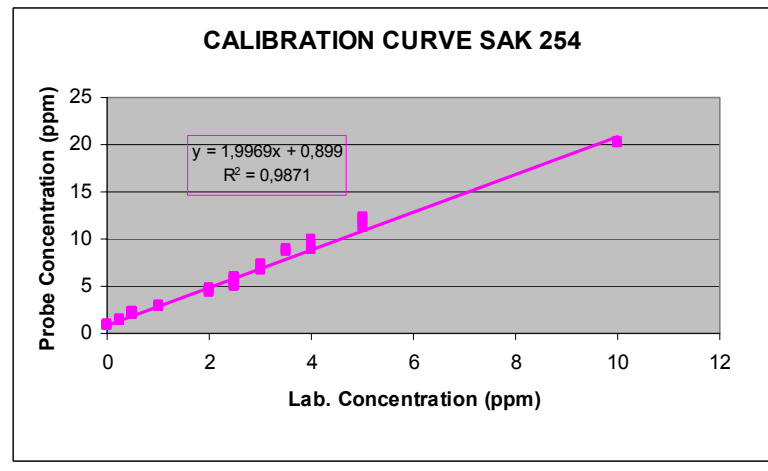

Figure 4: SAK254 calibration curve.

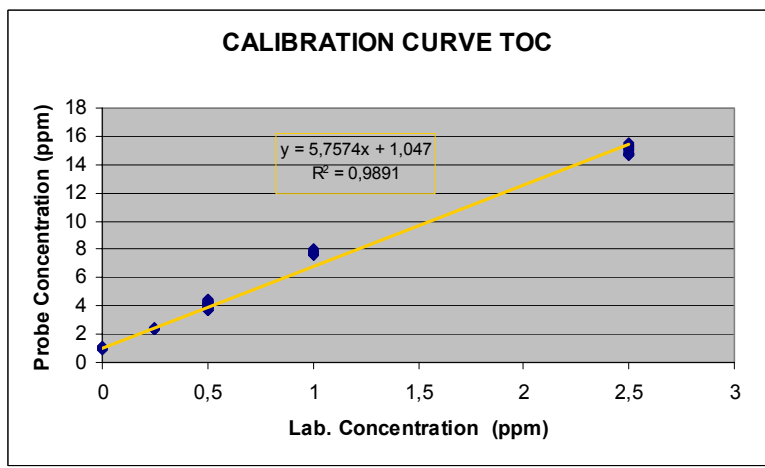

Figure 5: TOC calibration curve. 
The TOC could be detected down to $0,1 \mathrm{ppm}$ and the higher level of detection was $3 \mathrm{ppm}$. This shows that the probe results in high sensitivity on organic compound.

In particular, for the benzene, the lower limit of detection is $1 \mathrm{ppm}$ and the higher limit is 500 ppm; the water matrix doesn't affect the measures.

Regarding the Tri-methyl-benzene, it is extremely volatility in water, doesn't allow to correlate the absorption vs. concentration with high precision, but the probe is able to detect the presence of the substance also for low concentration.

\subsection{Application}

\subsubsection{Laboratory tests}

The UV/VIS spectrolyser was also tested in-situ, simulating a pollution release.

Since we are talking about drinking water, we decided to use as tracer an inert substance, like ascorbic acid (Vitamin C).

Moreover Vitamin $\mathrm{C}$ was identified as reliable absorbance indicator for the UV detection probe used in the EWWMS because it can simulate the presence of organic contaminant in the water.

As Figure 7 shows, ascorbic acid absorbs light in the range between 200 and $250 \mathrm{~nm}$; that is in a typical absorbance range of many organic compounds.

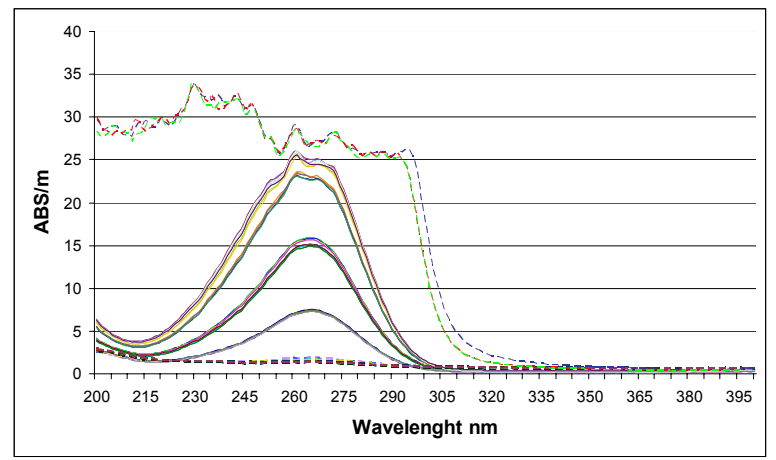

Figure 6: Typical UV spectra for ascorbic acid.

We did a proper calibration of each probes at different acid concentrations, in order to check the sensitivity of the system for different level of detection, and to compare the concentration of Vitamin $\mathrm{C}$ to the values given by the probes, and to establish the reproducibility of the measures. Concentrations of ascorbic acid in the range 0,1-10 ppm were tested.

The fingerprint of Vitamin $\mathrm{C}$ showed a clearly visible peak also for high concentrations, but in this case the value of the TOC parameter reached the saturation.

The value of SAK 254 and TOC increased linearly with the increasing of Vitamin $\mathrm{C}$ concentration. 
Also the nitrate concentration recorded by the probe was influenced; but the relation between the two values was not linear .

Figures 8 and 9 show the correlation between Vitamin C, TOC and SAK 254.

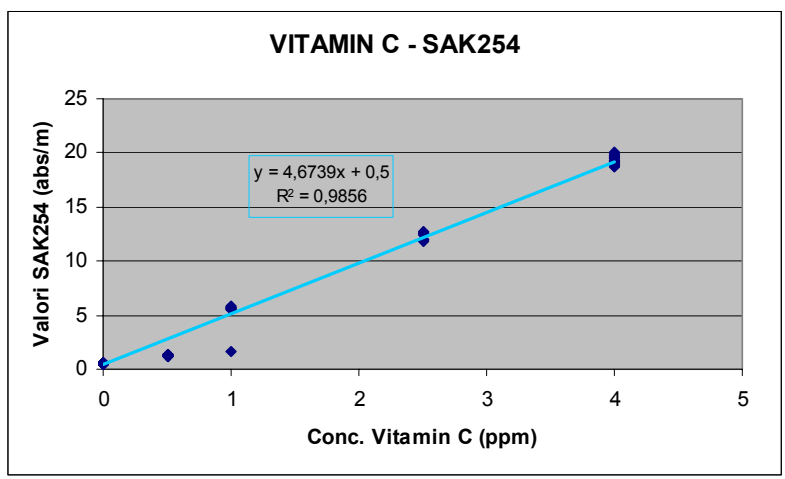

Figure 7: $\quad$ Correlation between Vitamin C and SAK254.

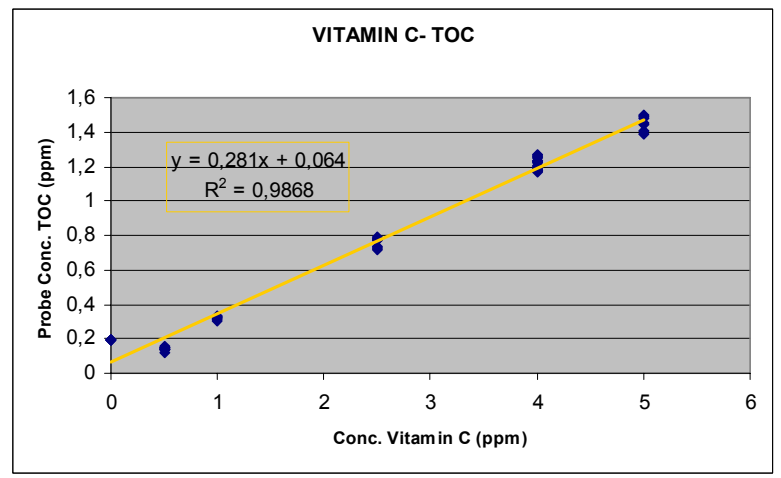

Figure 8: $\quad$ Correlation between Vitamin $\mathrm{C}$ and TOC.

After a long period of time to establish that the signal was stable and that the system could record clearly the difference in the water matrix, we spiked Vitamin $\mathrm{C}$ with a pre-set concentration.

Table 1 shows all the data necessary for the test.

During the test, the spiked concentration of ascorbic acid was kept at $0,5 \mathrm{mg} / \mathrm{l}$.

Figures 10 and 11 show the data recorded by the system during the test on the SYS1 station.

Data were registered every 30 seconds for SYS 1 and every 15 minutes for SYS 2. As Figures 12 and 13 show, data recorded in SYS 2 made visible a lower concentration than those in SYS1 because a dilution occurred in the thank before the SYS2 station. A shift in time of about 15 minute was also registered, due to the different retention time of the two systems. 
Table 1: $\quad$ Tracer test data.

\begin{tabular}{|c|l|}
\hline Spiked time & $1 \mathrm{~h}$ \\
\hline Vitamin C concentration & $0,5 \mathrm{mg} / \mathrm{l}$ \\
\hline Network Flow & $130 \mathrm{l} / \mathrm{s}$ \\
\hline Volume of solution & $300 \mathrm{I}$ \\
\hline Concentration in the tank & $780 \mathrm{mg} / \mathrm{l}$ \\
\hline Vitamin C flow & $5 \mathrm{l} / \mathrm{min}$ \\
\hline
\end{tabular}

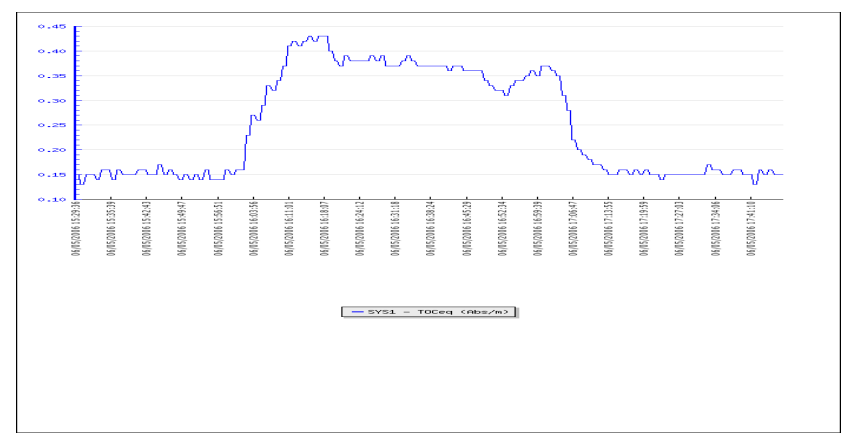

Figure 9: SAK254 data recorded by the system during the tracer test on SYS1.

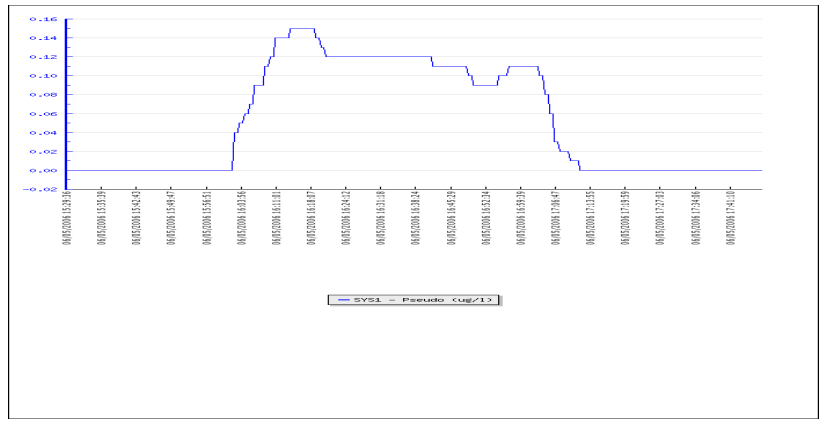

Figure 10: $\quad$ TOC data recorded by the system during the tracer test on SYS1. 


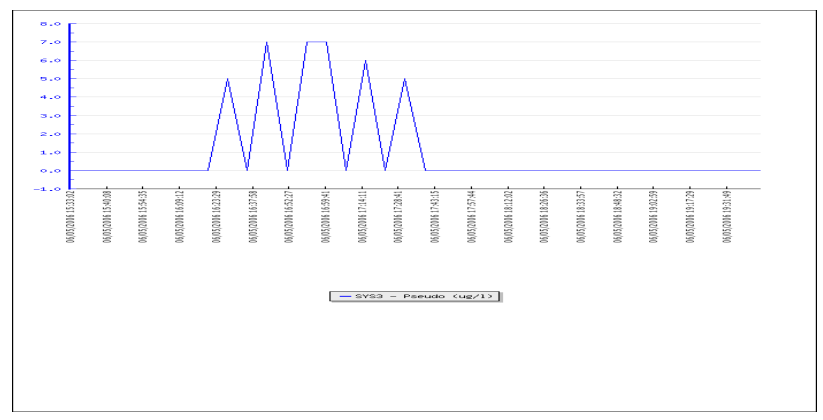

Figure 11: $\quad$ SAK254 data recorded by the system during tracer test on SYS2.

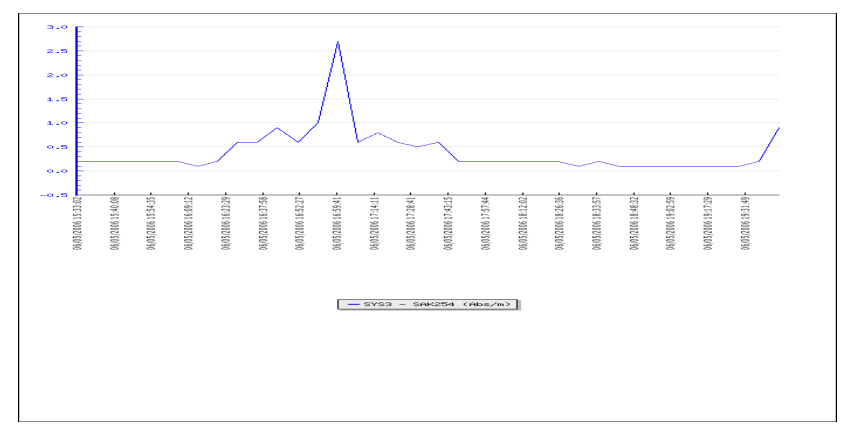

Figure 12: $\quad$ TOC data recorded by the system during tracer test on SYS2.

\section{Conclusion}

In order to develop a new early Warning System for contamination detection, a submergible UV/VIS spectrometer has been extensively tested in laboratory experiments, with the aim to check its sensitivity, its ability to identify and quantify specific contaminants and its rate of false positive/negative.

Laboratory and in situ tests have shown that the spectrolyser is easy to use and it provides robust results. It has high sensitivity and reproducibility especially for drinking water; it can also detect and monitoring the natural fluctuations in water composition. Moreover the probe is able to detect specific compounds and to quantify their concentration.

Even if the compound is unknown, the probe is able to detect him in drinking water, as not belonging to the natural fluctuation of the matrix. All the tests showed that the detection limits for all the parameters were very low. The spectral information for most compounds will not be enough for quantity identification, but parameters like TOC, DOC, SAK254 and NITRATE can be used to detect water contamination with unknown compounds. 
The entire system is able to detect an event of contamination, quickly and with high precision, and it has almost all the characteristics that an EWWS should have.

\section{References}

[1] Brosnan T.M. (1999). Early warning monitoring to detect hazardous events in water supplies. An ILSI Risk Science Institute Workshop Report.

[2] Gunatilaka A. and Dreher J (1996). Use of early warning systems as a tool for surface and ground water quality monitoring. Proc. IAWQ and IWSA Symp. on Metropolitan areas and Rivers, Rome. TS1 - River quality surveying and monitoring methods 2, 200-211.

[3] Ulitzur S. Lahav T. and Ulitzur N. (2002). A Novel and Sensitive Test for Rapid Determination of Water Toxicity. Environmental Toxicology Journal 17, 291-296.

[4] Clark, R. M., N. Adam, V. Atluri, M. Halem, E. Vowinkel, P. C. Tao, L. Cummings, and E. Ibrahim (2004). Developing an early warning system for drinking water security and safety. Pages 8.01-8.19 in L.W. Mays (ed.) Water supply systems security. New York, NY: McGraw-Hill Companies.

[5] Grayman, W. M., R. A. Deninger, and R. M. Males (2001). Design of early warning and predictive source water monitoring systems. Denver, CO: AWWA Research Foundation.

[6] Grayman, W. M., R. A. Deninger, and R. M. Clark (2002). Vulnerability of water supply to terrorist activities. CE News 14:34-38.

[7] Grayman, W. M., R. A. Deninger, R. M. Males, and R. W. Gullick (2004). Source water early warning systems. Pages 11.01-11.33 in L.W. Mays (ed.) Water supply systems security. New York, NY: McGraw-Hill and Companies.

[8] Langergraber, G., Weingartner, A., Fleischmann, N. (2004): Timeresolved delta spectrometry: A method to define alarm parameters from spectral data. Water Science \& Technology 50(11), 13-20.

[9] Langergraber, G., Fleischmann, N. and Hofstaedter, F. (2003). A multivariate calibration procedure for UV/VIS spectrometric quantification of organic matter and nitrate in wastewater. Wat. Sci. Tech., 47(2).

[10] Lee, J. Y., and R. A. Deininger (1992). Optimal locations of monitoring stations in water distribution systems. Journal of Environmental Engineers. 118(1): 4-16.

[11] Ostfeld, A, Kessler, A, Goldberg, I, A contaminant detection system for early warning in water distribution networks, (2004). Engineering Optimization, Vol. 36, No. 5, 525-538

[12] Jafrul Hasan, Stanley States, and Rolf Deininger, Safeguarding The Security Of Public Water Supplies Using Early Warning Systems: A Brief Review, (2004) Journal of Contemporary Water Research and Education, Issue 129, Pages 27-33. 